\title{
A Cryptic BCR-PDGFRB Fusion Resulting in a Chronic Myeloid Neoplasm With Monocytosis and Eosinophilia: A Novel Finding With Treatment Implications
}

\author{
Sanjeev Kumar Gupta, MD ${ }^{1, *}$; Nitin Jain, MD ${ }^{1, *} ;$ Guilin Tang, MD, PhD²; Andrew Futreal, PhD ${ }^{3} ; \mathrm{Sa} \mathrm{A}^{2}$ Wang, MD²; \\ Joseph D. Khoury, MD²; Richard K. Yang, MD, PhD²; Hong Fang, MD²; Keyur P. Patel, MD, PhD²; Rajyalakshmi Luthra, PhD²; \\ Mark Routbort, MD, PhD²; Bedia A. Barkoh, MS²; Wei Chen, MS²; Xizeng Mao, PhD³; Jianhua Zhang, PhD; \\ L. Jeffrey Medeiros, MD²; Carlos E. Bueso-Ramos, MD, $\mathrm{PhD}^{2}$; and Sanam Loghavi, $\mathrm{MD}^{2}$
}

\begin{abstract}
RNA-seq was used to identify the partner gene and confirm the presence of a BCR-PDGFRB fusion. Identification of this fusion product resulted in successful treatment and long-term remission of this myeloid neoplasm. Based on our results, we suggest that despite current WHO recommendations, screening for PDGFRB rearrangement in cases of leukocytosis with eosinophilia and no other etiologic explanation is necessary, even if the karyotype is normal.
\end{abstract}

J Natl Compr Canc Netw 2020;18(10):1300-1304 doi: $10.6004 /$ jnccn.2020.7573

Departments of ${ }^{1}$ Leukemia, ${ }^{2}$ Hematopathology, and ${ }^{3}$ Genomic Medicine, The University of Texas MD Anderson Cancer Center, Houston, Texas.

*These authors contributed equally.

\section{Background}

Myeloid and lymphoid neoplasms with eosinophilia and gene rearrangement constitute a distinct group of hematologic neoplasms in the current WHO classification for hematopoietic neoplasms. ${ }^{1}$ Included in this category are neoplasms harboring abnormal gene fusions involving PDGFRA, PDGFRB, and FGFR1 or PCM1-JAK2. These fusions result in constitutive activation of respective tyrosine kinases that can be targeted using specific kinase inhibitors. There are multiple recognized partner genes for PDGFRA, PDGFRB, FGFR1, and JAK2. PDGFRB located at chromosome $5 \mathrm{q} 32$ has $>25$ known fusion partners, with ETV6 the most common..$^{2-8}$ Although PDGFRA rearrangements often can be cryptic and not readily observed on routine karyotyping studies, ${ }^{9,10}$ PDGFRB rearrangements are believed to be nearly always visible on routine karyotype. and the WHO textbook specifically states that "molecular analysis for PDGFRB is not necessary for myeloid neoplasms with eosinophilia if routine karyotypic studies do not reveal a $5 q$ abnormality." 1

This report describes a myeloid neoplasm presenting with morphologic features of chronic myelomonocytic leukemia with eosinophilia harboring a cryptic and novel $B C R-P D G F R B$ fusion. Discovery of this fusion led to successful treatment with imatinib, a tyrosine kinase inhibitor. To our knowledge, this is the first report of a $B C R-P D G F R B$ fusion in a myeloid neoplasm associated with eosinophilia.

\section{Methods}

Next-generation sequencing (NGS) was performed on DNA obtained from the bone marrow (BM) aspirate using a whole exome panel of 28 genes commonly associated with myeloid neoplasms, as described previously. ${ }^{11}$ 
Conventional chromosomal analysis was performed on G-banded metaphases prepared from unstimulated 48-hour BM aspirate cultures using standard techniques; 20 metaphases were analyzed

Fluorescence in situ hybridization (FISH) was performed using a PDGFRB dual-color breakapart probe set (Cytocell Ltd).

RNA-seq analysis was performed using $700 \mathrm{ng}$ of total RNA and a TruSeq RNA Sample Preparation Kit v2 (Illumina). The library was enriched selectively by 12 cycles of PCR followed by size selection per the manufacturer's protocol. The resulting size-fractionated library was sequenced using a 75-bp short-read paired-end reads protocol on an Illumina HiSeq 2000 Sequencing System. The resultant BCL files were demultiplexed and converted to fastq files using Illumina's Consensus Assessment of Sequence and Variation (CASAVA) tool. After standard quality control using RNA-SeQC, fastq files were processed using an integrative analysis of multiple fusion detection methods with hg19 as the reference genome. To increase specificity, 3 fusion detection tools-TopHat-Fusion, MapSplice, and FusionMap-were applied; only fusions detected by at least 2 tools were considered positive. ${ }^{12-14}$

\section{Results}

The patient was a 26 -year-old man who presented with a 2-week history of fatigue, sore throat, night sweats, muscle aches, and weight loss. A diagnostic workup was initiated with a clinical consideration of acute leukemia in view of the short symptomatic history and pathology report from another institution indicating a "packed" bone marrow. Physical examination of the patient showed splenomegaly. Renal function, liver function, and other biochemical parameters, including serum lactate dehydrogenase levels, were within normal limits. A CBC count revealed anemia (hemoglobin level, $11.8 \mathrm{~g} / \mathrm{dL}$; reference, 13.5-17.5 g/dL), leukocytosis (WBC count, $37.6 \times 10^{9} / \mathrm{L}$; reference, $4-11 \times 10^{9} / \mathrm{L}$ ), and a normal platelet count $\left(152 \times 10^{9} / \mathrm{L}\right.$; reference, $\left.150-450 \times 10^{9} / \mathrm{L}\right)$. The differential leukocyte count showed monocytosis (47\%) and eosinophilia (15\%). BM examination showed hypercellular $(\sim 95 \%)$ BM with myelomonocytic hyperplasia and eosinophilia including increased eosinophilic
A

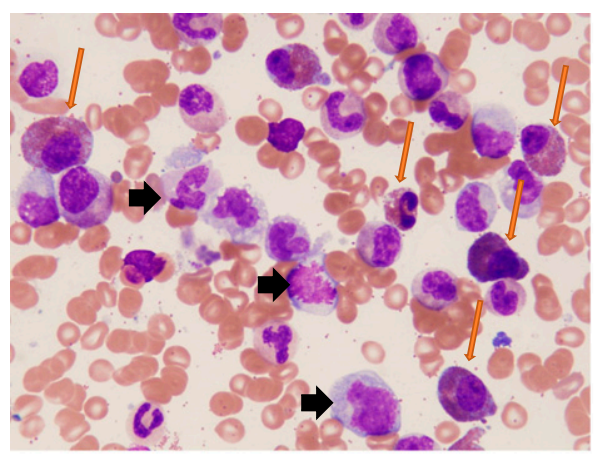

C

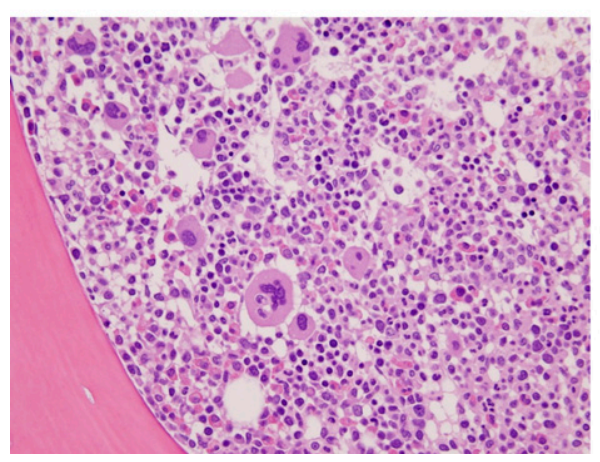

B

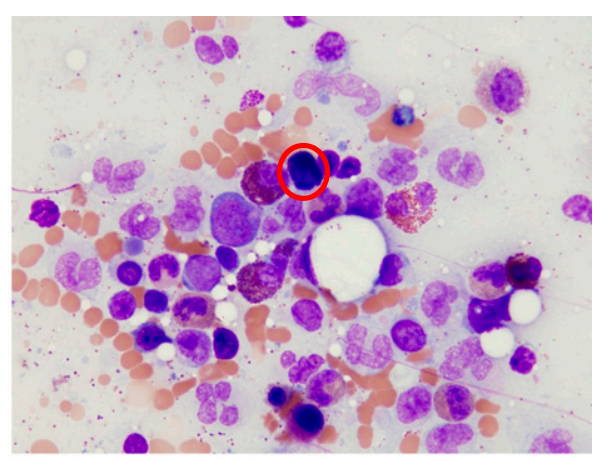

D

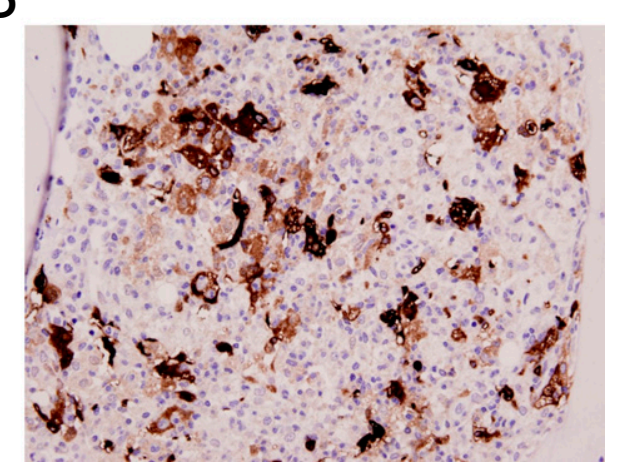

Figure 1. Bone marrow aspirate smear showing $(\mathrm{A})$ increased monocytes with atypical morphology (black arrows), eosinophilic precursors (orange arrows), and (B) occasional morphologically unremarkable mast cells (red circle) (Wright-Giemsa, original magnification $\times 1,000)$. (C) Bone marrow trephine biopsy specimen showing hypercellular bone marrow with marked myelomonocytic hyperplasia with increased eosinophils and increased atypical megakaryocytes (some with separated nuclear lobes), the latter showing emperipolesis (hematoxylin-eosin, original magnification $\times 400$ ). (D) A mast cell tryptase stain shows increased mast cells; however, no abnormal aggregates were identified (immunohistochemistry with hematoxylin counterstain, original magnification $\times 400)$. 
myelocytes and metamyelocytes, erythroid hypoplasia, and no significant increase in blasts. Megakaryocytes were mildly increased but did not form distinct clusters; they had predominantly unremarkable morphology and showed frequent emperipolesis (Figure 1A-C). Mast cells were increased but did not form abnormal aggregates (Figure 1D). Ring sideroblasts were not identified. Flow cytometric immunophenotypic analysis showed a small $(0.2 \%)$ population of myeloid blasts without immunophenotypic aberrancies. Monocytes were increased (26\%) and showed aberrant CD56 expression. A small population of mast cells with aberrant expression of CD25 and CD30 was also present. Conventional cytogenetics showed a diploid male karyotype, 46,XY[20] (Figure 2A). FISH was negative for $B C R-A B L 1$. Microarray-based comparative genomic hybridization (array CGH) revealed no chromosomal copy number alterations. NGS did not reveal any mutations. Because of the presence of eosinophilia, additional FISH testing for PDGFRA, PDGFRB, and FGFR1 was performed, revealing a $P D G F R B$ rearrangement in $55 \%$ of the interphase nuclei analyzed (Figure 2B); PDGFRA and FGFR1 were intact. A mapback FISH analysis on a G-banded metaphase showed that 3'PDGFRB (red) had translocated to a morphologically normal chromosome $22 \mathrm{q}$ and that 5'PDGFRB (green) had remained on chromosome 5q (Figure 2C). The RNA-seq FusionMap caller identified 2 unique fusion reads that defined an in-frame fusion joining exon 13 of PDGFRB on chromosome 5 (149504394; hg19) to exon 1 of $B C R$ on chromosome 22 (23524426; hg19). The MapSplice caller further confirmed the same BCR-PDGFRB with similar breakpoints, with 3 unique read counts and 1 paired-read. The resultant fusion product was formed by the first 426 residues of $B C R$ fused to the last 505 residues of $P D G F R B$ containing the oligomerization domain and serine threonine kinase domain of $B C R$ and the kinase domain of PDGFRB (Figure 2D). In light of these findings, a
A
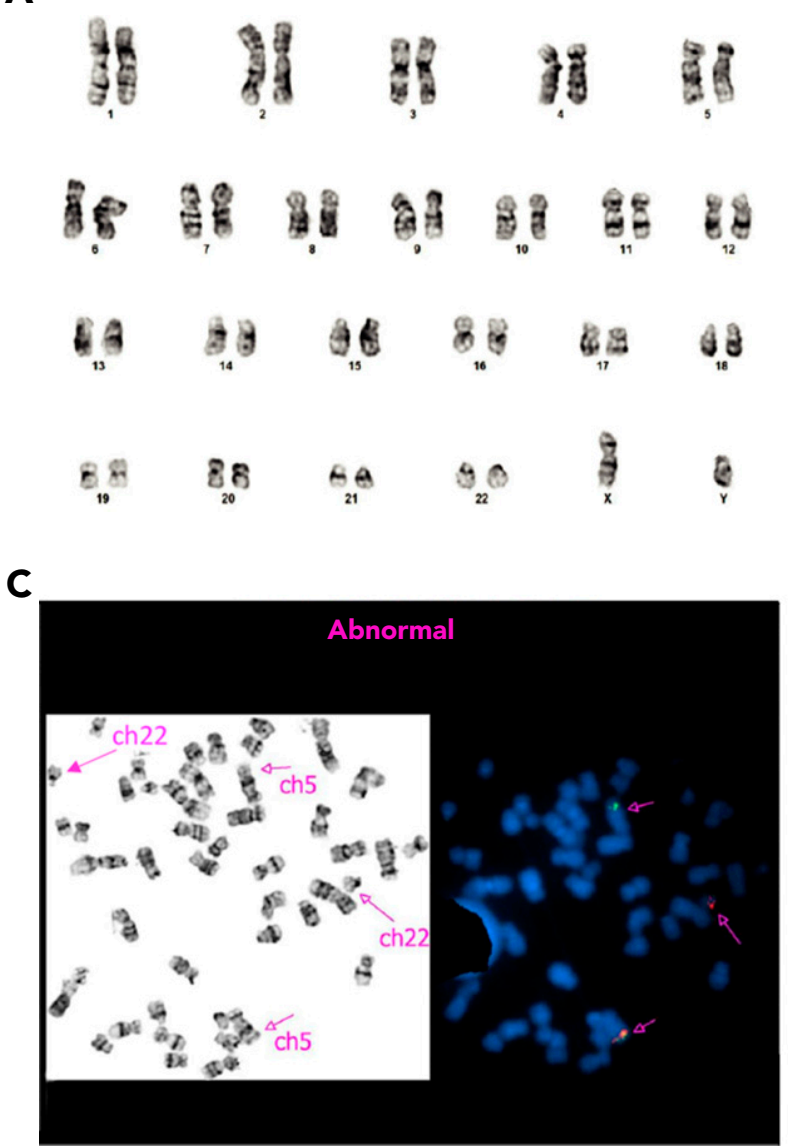

B
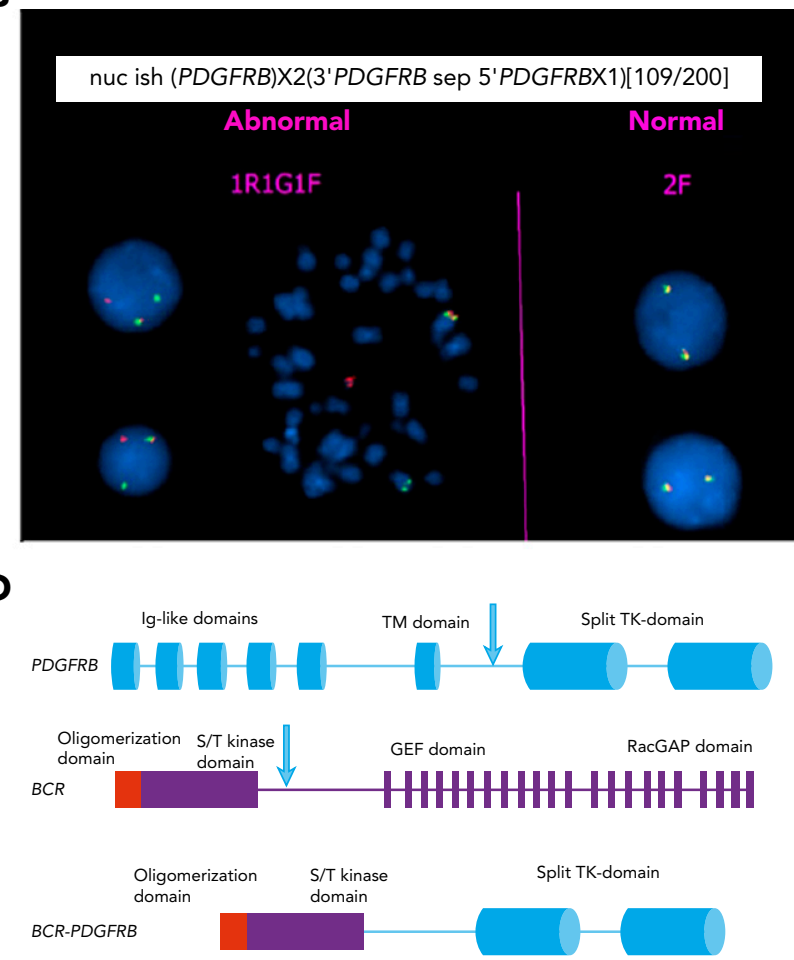

donor sequence: 5'-GAGGGCGCCTTCCATGGAGACGCAG-3' at the 3'end of exon 1 of BCR acceptor sequence: $3^{\prime}$-AAGGCCCCAGAGCCGAGGGTGCGTC-5' at the 5 'end of exon 13 of PDGFRB

Figure 2. (A) Chromosomal analysis showed a diploid male karyotype 46,XY. (B) FISH analysis using a Cytocell dual-color breakapart PDGFRB probe showed split signals, indicating PDGFRB rearrangement. Normal pattern: 2 fusion (2F) yellow signals; abnormal pattern: 1 red, 1 green, 1 fusion (1R1G1F) signals. (C) Mapback FISH analysis on a G-banded metaphase with a Cytocell dual-color breakapart PDGFRB probe revealed 3'PDGFRB (red) on chromosome 22 and 5'PDGFRB (green) on chromosome 5. (D) Schematic representation of the BCR-PDGFRB fusion product with donor and acceptor sequences.

Abbreviations: ch, chromosome; FISH, fluorescence in situ hybridization; Ig, immunoglobulin; S/T, serine/threonine; TK, tyrosine kinase; TM, transmembrane. 
retrospective review of the routine karyotype was performed that confirmed the alteration to be cryptic.

\section{Discussion}

Myeloid neoplasms with eosinophilia and PDGFRB rearrangement have been described more commonly in men (2:1 ratio) over a wide age range (8-72 years), with median age in the late 40s. The hematologic presentation in cases of myeloid neoplasms with PDGFRB rearrangement is most often that of chronic myelomonocytic leukemia, usually with eosinophilia, as was seen in this case. ${ }^{6,15}$ The BCR-PDGFRB we describe is a novel fusion, not yet documented in the published literature. The fusion retained the oligomerization and serine threonine kinase domains of $B C R$, supporting constitutive activation of the retained tyrosine kinase domain of PDGFRB. This hypothesis is supported by the prompt, effective, and long-lasting control of leukocytosis and eosinophilia through treatment with imatinib in this patient. The patient was treated with imatinib, $400 \mathrm{mg}$ daily, which resulted in complete response with normalization of blood counts and disappearance of eosinophilia within 3 months. A follow-up BM sample performed 3 months after initial diagnosis was morphologically unremarkable and negative for PDGFRB rearrangement by FISH. At the time of writing, the patient is undergoing regular follow-up and continues to be experiencing disease remission after 3 years of therapy.

In addition to showing a novel fusion product, this case shows a clinically critical point: that screening for $P D G F R B$ rearrangement in cases of leukocytosis with eosinophilia and no other etiologic explanation may be helpful and potentially lifesaving, even if the routine karyotype is diploid.

Due to the large number of potential fusion partner genes, molecular analysis, namely RNA-seq, can be helpful in identifying a partner gene once rearrangement is detected by FISH. In addition to confirming the diagnosis, molecular characterization of the fusion at diagnosis using RT-PCR or RNA sequencing will provide a potential target for monitoring treatment response and measuring residual disease. The presence of immunophenotypically abnormal mast cells as seen in this case is also a helpful clue to assess for PDGFRB alterations, because these cases frequently harbor abnormal mast cell populations, despite not meeting diagnostic criteria for systemic mastocytosis. ${ }^{1,16}$

It is useful to perform immunohistochemistry for mast cells tryptase and/or CD117 to exclude systemic mastocytosis. Unlike systemic mastocytosis, the mast cells described in this case do not form dense aggregates and are not morphologically abnormal (spindled); furthermore, a KIT D816V mutation was not detected. This case also highlights the importance of a systematic workup for potential recurrent gene rearrangements associated with myeloid neoplasms with eosinophilia. Early diagnosis of the underlying molecular aberration in myeloid neoplasms with eosinophilia will help in the timely assignment of appropriate targeted therapy and reducing the probability of potential tissue damage associated with eosinophilia. In some patients, timely identification might help induce a remission that may permit allogeneic stem cell transplant therapy. ${ }^{10}$ This case also highlights the utility of a stepwise approach in using PDGRB FISH for accurate diagnosis of myeloid neoplasms with eosinophilia that do not have evidence of 5q31-33 alterations on routine karotypic analysis, in which the presence of eosinophilia cannot be attributed to other molecular or cytogenetic alterations, such as $B C R-A B L 1$ translocation or alterations of PDGFRA. ${ }^{17}$ Currently FISH is considered to be more practical and readily available in most commercial and academic settings. Although more advanced technologies such as RNA-seq are useful in identifying unknown gene partners in these neoplasms, as shown in our case, they are not readily accessible outside of the research setting, and therefore we do not currently recommend their routine use in the workup of such cases.

Although limited by its observational nature, this case does highlight a potential pitfall in the diagnosis of myeloid neoplasms with eosinophilia, and supports the algorithmic approach recommended by Fang et $\mathrm{al}^{17}$ in their recent publication describing the systematic use of PDGFRB FISH in such neoplasms.

\section{Conclusions}

This report presents the case of a myeloid neoplasm with monocytosis and eosinophilia harboring a novel and cryptic PDGFRB rearrangement leading to a $B C R-P D G F R B$ fusion. Identification of this fusion protein resulted in successful treatment and long-term sustained remission of this myeloid neoplasm.

\footnotetext{
Submitted February 6, 2020; accepted for publication April 6, 2020.
}

Author contributions: Study concept and design: Jain, Bueso-Ramos, Loghavi. Patient treatment: Jain. Pathologic workup and case characterization: Tang, Wang, Khoury, Futreal, Bueso-Ramos, Medeiro, Patel. RNA-seq studies and data analysis: Futreal, Barkoh, Chen, Mao, Zhang. Manuscript preparation: All authors.

Disclosures: The authors have disclosed that they have not received any financial consideration from any person or organization to support the preparation, analysis, results, or discussion of this article

Correspondence: Sanam Loghavi, MD, Department of Hematopathology, The University of Texas MD Anderson Cancer Center, 1515 Holcombe Boulevard, 0072, Houston, TX 77030. Email: sloghavi@mdanderson.org 


\section{References}

1. Bain BJ, Horny HP, Arber DA, et al. Myeloid/lymphoid neoplasms with eosinophilia and rearrangement of PDGFRA, PDGFRB or FGFR1, or with PCM1-JAK2. In: Swerdlow SH, Campo E, Harris NL, et al, eds. WHO Classification of Tumours of Haematopoietic and Lymphoid Tissues. Lyon, France: International Agency for Research on Cancer (IARC); 2017:72-79.

2. Bain BJ, Fletcher SH. Chronic eosinophilic leukemias and the myeloproliferative variant of the hypereosinophilic syndrome. Immunol Allergy Clin North Am 2007;27:377-388.

3. Steer EJ, Cross NC. Myeloproliferative disorders with translocations of chromosome 5q31-35: role of the platelet-derived growth factor receptor beta. Acta Haematol 2002;107:113-122.

4. David M, Cross NC, Burgstaller S, et al. Durable responses to imatinib in patients with PDGFRB fusion gene-positive and BCR-ABL-negative chronic myeloproliferative disorders. Blood 2007;109:61-64.

5. Bain BJ. Myeloid and lymphoid neoplasms with eosinophilia and abnormalities of PDGFRA, PDGFRB or FGFR1. Haematologica 2010;95 696-698.

6. Vega F, Medeiros LJ, Bueso-Ramos CE, et al. Hematolymphoid neoplasms associated with rearrangements of PDGFRA, PDGFRB, and FGFR1. Am J Clin Pathol 2015;144:377-392.

7. Curtis CE, Grand FH, Waghorn K, et al. A novel ETV6-PDGFRB fusion transcript missed by standard screening in a patient with an imatinib responsive chronic myeloproliferative disease. Leukemia 2007;21: 1839-1841.

8. Crescenzi B, La Starza R, Nozzoli C, et al. Molecular cytogenetic findings in a four-way $t(1 ; 12 ; 5 ; 12)(p 36 ; p 13 ; q 33 ; q 24)$ underlying the ETV6-PDGFRB fusion gene in chronic myelomonocytic leukemia. Cancer Genet Cytogenet 2007;176:67-71.
9. Hu Z, Boddu PC, Loghavi S, et al. A multimodality work-up of patients with hypereosinophilia. Am J Hematol 2018;93:1337-1346.

10. Shah S, Loghavi S, Garcia-Manero G, et al. Discovery of imatinibresponsive FIP1L1-PDGFRA mutation during refractory acute myeloid leukemia transformation of chronic myelomonocytic leukemia. J Hemato Oncol 2014;7:26.

11. Quesada AE, Hu Z, Routbort MJ, et al. Mixed phenotype acute leukemia contains heterogeneous genetic mutations by next-generation sequencing. Oncotarget 2018;9:8441-8449.

12. Ge H, Liu K, Juan T, et al. FusionMap: detecting fusion genes from nextgeneration sequencing data at base-pair resolution. Bioinformatics 2011 ; 27:1922-1928.

13. Wang K, Singh D, Zeng Z, et al. MapSplice: accurate mapping of RNA-seq reads for splice junction discovery. Nucleic Acids Res 2010;38:e178.

14. Kim D, Salzberg SL. TopHat-fusion: an algorithm for discovery of novel fusion transcripts. Genome Biol 2011;12:R72.

15. Patnaik MM, Lasho $T L$, Finke $C M$, et al. Targeted next generation sequencing of PDGFRB rearranged myeloid neoplasms with monocytosis. Am J Hematol 2016;91:E12-E14.

16. Walz C, Metzgeroth G, Haferlach C, et al. Characterization of three new imatinib-responsive fusion genes in chronic myeloproliferative disorders generated by disruption of the platelet-derived growth factor receptor beta gene. Haematologica 2007;92:163-169

17. Fang $H$, Tang $G$, Loghavi $S$, et al. Systematic use of fluorescence in situ hybridization (FISH) and clinicopathological features in the screening of PDGFRB rearrangements of patients with myeloid/lymphoid neoplasms [published online February 21, 2020]. Histopathology, doi: 10.1111/ his.14097 